\title{
Advanced Turbine System (ATS) Program Conceptual Design and Product Development
}

\author{
Quarterly Report \\ September 1 - November 30, 1995
}

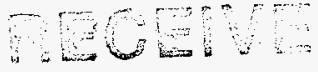

M00007

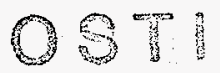

Work Performed Under Contract No.: DE-AC21-93MC30244

For

U.S. Department of Energy

Office of Fossil Energy

Morgantown Energy Technology Center

P.O. Box 880

Morgantown, West Virginia 26507-0880

By

General Electric Company

Power Generation Engineering

1 River Road

Schenectady, New York 12345

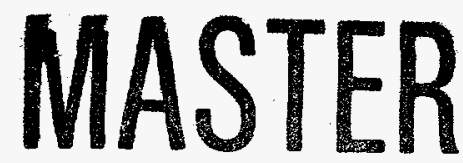

$\mathrm{Ht}$ 


\section{Disclaimer}

This report was prepared as an account of work sponsored by an agency of the United States Government. Neither the United States Government nor any agency thereof, nor any of their employees, makes any warranty, express or implied, or assumes any legal liability or responsibility for the accuracy, completeness, or usefulness of any information, apparatus, product, or process disclosed, or represents that its use would not infringe privately owned rights. Reference herein to any specific commercial product, process, or service by trade name, trademark, manufacturer, or otherwise does not necessarily constitute or imply its endorsement, recommendation, or favoring by the United States Government or any agency thereof. The views and opinions of authors expressed herein do not necessarily state or reflect those of the United States Government or any agency thereof. 


\section{DISCLAIMER}

Portions of this document may be illegible in electronic image products. Images are produced from the best available original document. 


\title{
REPORTING PERIOD 9/1/95 - 11/30/95
}

\author{
Table of Contents
}

Executive Summary

ATS Phase 2 Program Status

Task 4 - Conversion to a Coal Fueled ATS

Task 8 - Design and Test of Critical Components
TAB 1

TAB 2

TAB 3

TAB 4 


\subsection{EXECUTIVE SUMMARY}

\section{GE Advanced Turbine Systems Program}

GE has achieved a leadership position in the worldwide gas turbine industry in both industrialutility markets and in aircraft engines. This design and manufacturing base plus our close contact with the users provides the technology for creation of the next generation advanced power generation systems for both the industrial and utility industries. GE has been active in the definition of advanced turbine systems for several years. These systems will leverage the technology from the latest developments in the entire GE gas turbine product line. These products will be USA-based in engineering and manufacturing and are marketed through GE Power Systems.

Achieving the Advanced Turbine Systems (ATS) goals of $60 \%$ efficiency, single-digit NOx, and 10\% electric power cost reduction imposes competing characteristics on the gas turbine system. Two basic technical issues arise from this. The turbine inlet temperature of the gas turbine must increase to achieve both the efficiency and cost goals. However, higher temperatures move in the direction of increased NOx emissions. Improved coatings and other materials technologies along with creative combustor design can result in solutions whcih will achieve the ultimate goal.

GE's view of the market, in conjunction with the industrial and utility objectives, requires the development of Advanced Gas Turbine Systems which encompass two potential products: a new aeroderivative combined-cycle system for the industrial market, and a combined-cycle system for the utility sector that is based on an advanced frame machine.

The GE Advanced Gas Turbine Development program is focused on two specific products:

1. A $70 \mathrm{MW}$ class industrial gas turbine based on the GE90 core technology utilizing an innovative air cooling methodology;

2. A $200 \mathrm{MW}$ class utility gas turbine based on an advanced GE heavy-duty machine utilizing advanced cooling and enhancement in component efficiency.

Both of these activities require the identification and resolution of technical issues critical to achieving ATS goals. The emphasis for the industrial ATS will be placed upon innovative cycle design and low emission combustion. The emphasis for the utility ATS will be placed on developing a technology base for advanced turbine cooling, while utilizing demonstrated and planned improvements in low emission combustion. Significant overlap in the development programs will allow common technologies to be applied to both products. GE Power Systems is solely responsible for offering GE products for the industrial and utility markets. The GE ATS program will be managed fully by this 
organization, with core engine technology being supplied by GE Aircraft Engines (GEAE), and fundamental studies supporting both product developments being conducted by GE Corporate Research and Development (CRD). GE's worldwide experience in commercialization of these products will ensure that the ATS program can proceed to the marketplace. 


\subsection{ATS PHASE 2 PROGRAM STATUS}

GE Power Generation has continued work on the ATS Phase 2 contract. At the request of DOE/Morgantown, an expanded scope of work was proposed, and has been accepted. The tasks in the expanded work scope are shown in Table 2-1. The period of performance for Phase 2 has been extended from 8/25/93 - 9/15/95 to 8/25/93 - 3/31/96.

The status of Task 1 through Task 8 is given in Table 2-2, and graphic representations are shown in Figures 2.1 and 2.2. The ATS Phase 2 program is on schedule for completion per the contract.

\section{Table 2-1}

1 Preliminary Design 6

2 Coolant Purity $\quad 8.1$

3 Advanced Seals $\quad 8.4$

4 Rotational Heat Transfer $\quad 8.6$

5 Effect of Hot Fuel on Combustion Dynamics $\quad 8.7$

6 Rotor Materials Coolant Compatibility 8.2

6P Nozzle Cascade Prel. Test \& Facility Qualification $\quad 8.7$

7 TBC Testing and Analysis $\quad 8.3$

8 LCF Life and Crack Propagation $\quad 8.3$

9 Turbulence Intensity Probes $\quad 8.7$

12 Bond Coats for Improved TBC Thermal Cycle Life 8.3

13 Rotational Heat Transfer-Wheelspace Cooling 8.6

15 Short Liner $\quad 8.7$

16 Turbulent Heat Transfer-Static Components 8.7

18 Critical TBC Properties $\quad 8.3$

19 Increased MACH Number Diffuser Design $\quad 8.7$

20 Transition Piece Design Tools 8.7 
Table 2-2

\section{Status Of Tasks 1 Through 8}

\section{Task 1: Project Plan}

The Project Plan, which develops and documents the overall work scope for the ATS Phase 2 Statement of Work Tasks 2 through 8, was submitted and approved by DOE.

\section{Task 2: Information Required for National Environmental Policy Act (NEPA)}

The NEPA report, which covers environmental impact assessments of the test facility sites, was submitted and approved by DOE.

\section{Task 3: Selection of a Natural Gas Fired Advanced Turbine System (GFATS)}

\section{Task 3A: Selection of an Industrial Natural Gas Fired Advanced Turbine System (GFATS)}

The industrial GFATS cycle selection was completed last year, and was described in the last yearly report. The cycle selected is based on the GE90 aero engine, with intercooling and turbocooling. This engine also meets all of the ATS utility machine performance goals. A summary is given in the Annual Report.

\section{Task 3B: Selection of a Utility Natural Gas Fired Advanced Turbine System (GFATS)}

The utility GFATS cycle section was completed last year. A summary is included in the Annual Report. The cycle selected features closed-loop steam cooling in the first and second stages of its four stage turbine, $2600^{\circ} \mathrm{F} / 1430^{\circ} \mathrm{C}$ class of firing temperatures, a higher pressure ratio compressor derived from the CF6-80C2 aircraft engine, and an advanced dry low NOx combustion system. This machine meets all of the ATS performance goals.

\section{Task 4: Conversion to a Coal Fueled ATS (CFATS)}

\section{Task 4A - Industrial CFATS}

Four gasifier systems were reviewed for constituent concentrations, coal gas heating value, required engine bleed flow, and the fuel flow required to maintain the required power level. A summary is given in the TAB 3 section. 


\section{Task 4B - Utility CFATS}

The Utility CFATS analysis is in progress. Particular emphasis has been placed on integration with the Shell-based gasification system. A summary is given in the TAB 3 section.

\section{Task 5: Market Study}

The market study was performed with cycle information developed in Task 3A and Task 3B. This task has been completed.

\section{Task 6: System Definition and Analysis}

The Industrial ATS (GE90 based) preliminary design was completed. A summary is contained in the Annual Report. The Utility ATS $(7 \mathrm{H})$ machine preliminary design task is completed. A status report is contained in the Annual Report section.

\section{Task 7: Integrated Program Plan}

No progress on this Task during reporting period. Task will be performed with information developed in Task 3 and Task 6 for both ATS designs.

\section{Task 8: Design and Test of Critical Components}

Work proceeded on the experimental evaluation that will enable implementation of advances in several areas critical to the Industrial and Utility ATS systems. Seven major subtasks (listed below) comprise this task. Each work effort is reported individually in the $\mathrm{TAB} 7$ section.

Task 8.1 Particulate Flow Deposition

Task 8.2 Particle Centrifugal Sedimentation

Task 8.3 TBC Mechanical Test and Analysis

Task 8.4 Advanced Seal Technology

Task 8.5 Enhanced Impingement Heat Transfer

Task 8.6 Rotating Heat Transfer

Task 8.7 Turbine Inlet Nozzle Heat Transfer 


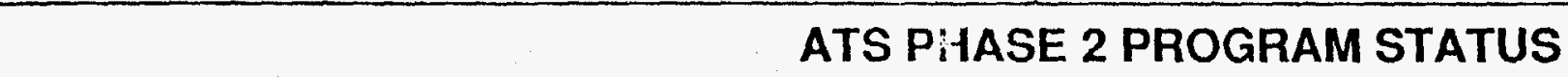

\begin{tabular}{|c|c|c|c|c|c|c|c|c|c|c|c|c|}
\hline \multirow[b]{2}{*}{ ID } & \multirow[b]{2}{*}{ Task Name } & \multicolumn{2}{|l|}{93} & \multicolumn{4}{|c|}{1994} & \multicolumn{4}{|c|}{1995} & \multirow[b]{2}{*}{ Otr 1} \\
\hline & & Qtr 3 & Qtr 4 & Qtr 1 & Qut 2 & Q13 3 & Qtr 4 & Qtr 1 & atr 2 & Qtr 3 & Qtr 4 & \\
\hline 1 & Task 1 - Project Plan & & & & & & & & & & & \\
\hline 2 & & & & & & & & & & & & \\
\hline 3 & Task 2 - NEPA Information & & & & & & & & & & & \\
\hline 4 & & & & & & & & & & & & \\
\hline 5 & Task 3 - Gas Fired ATS Selection & & & & & & & & & & & \\
\hline 6 & & & & & & & & & & & & \\
\hline 7 & Task 4 - Conversion to Coal & & & & & & & & & & & \\
\hline 8 & & & & & & & & & & & & \\
\hline 9 & Task 5 - GFATS Market Study & & & & & & & & & & & \\
\hline 10 & & & & & & & & & & . & & \\
\hline $\begin{array}{ll}11 \\
+\end{array}$ & Task 6 - System Definition \& Analysis & & & & & & & & & & & \\
\hline 12 & & & & & & & & & & & & \\
\hline 13 & Task 7 - Integrated Program Plan & & & & & & & & & & & \\
\hline 14 & & & & & & & & & & & & \\
\hline 15 & Task 8 - Design/Test Critical Components & & & & & & & & & & & \\
\hline
\end{tabular}

Figure 2-1 


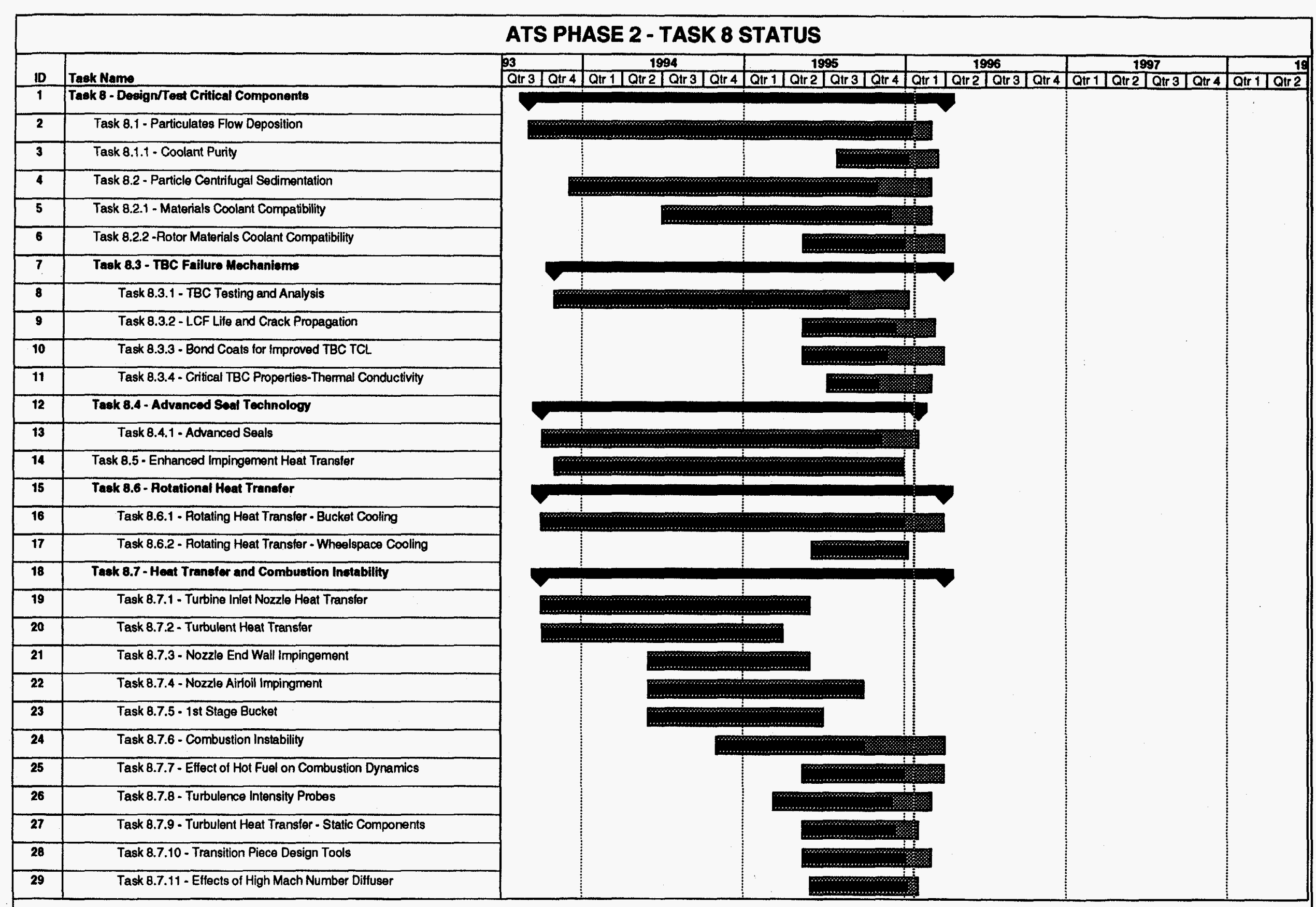

Figure 2-2 


\section{TASK 4 - CONVERSION TO A COAL FUELED ATS (CFATS)}

\section{Description}

For both Industrial and Utility systems, select a coal fueled cycle based on adaptation of the GFATS and identify the work required to modify the GFATS to perform optimally in the CFATS.

\section{Objectives}

Select a CFATS that will be most marketable after the year 2005 by best using the basic GFATS design while optimizing the use of coal gas fuel. Define the changes that are required and identify necessary provisions in the GFATS design to accept those changes to minimize future conversion costs to coal fuel. Define development work necessary to promote successful operation of the CFATS.

\section{Progress}

\section{Task 4A - Industrial CFATS}

Four gasifier systems have been reviewed for constituent makeup, coal gas heating value, required engine bleed flow, and the required fuel flow to maintain the required power level. The Shell system has been selected as the coal gas configuration to be studied under the ATS Program. This effort is ongoing. The gasifier/gas turbine interaction is shown by the schematic in Figure 3-1. Table 3-1 outlines the required engine and gasifier flows of the four systems reviewed and outlines the unique requirements of the Shell system. 

Table 3-1

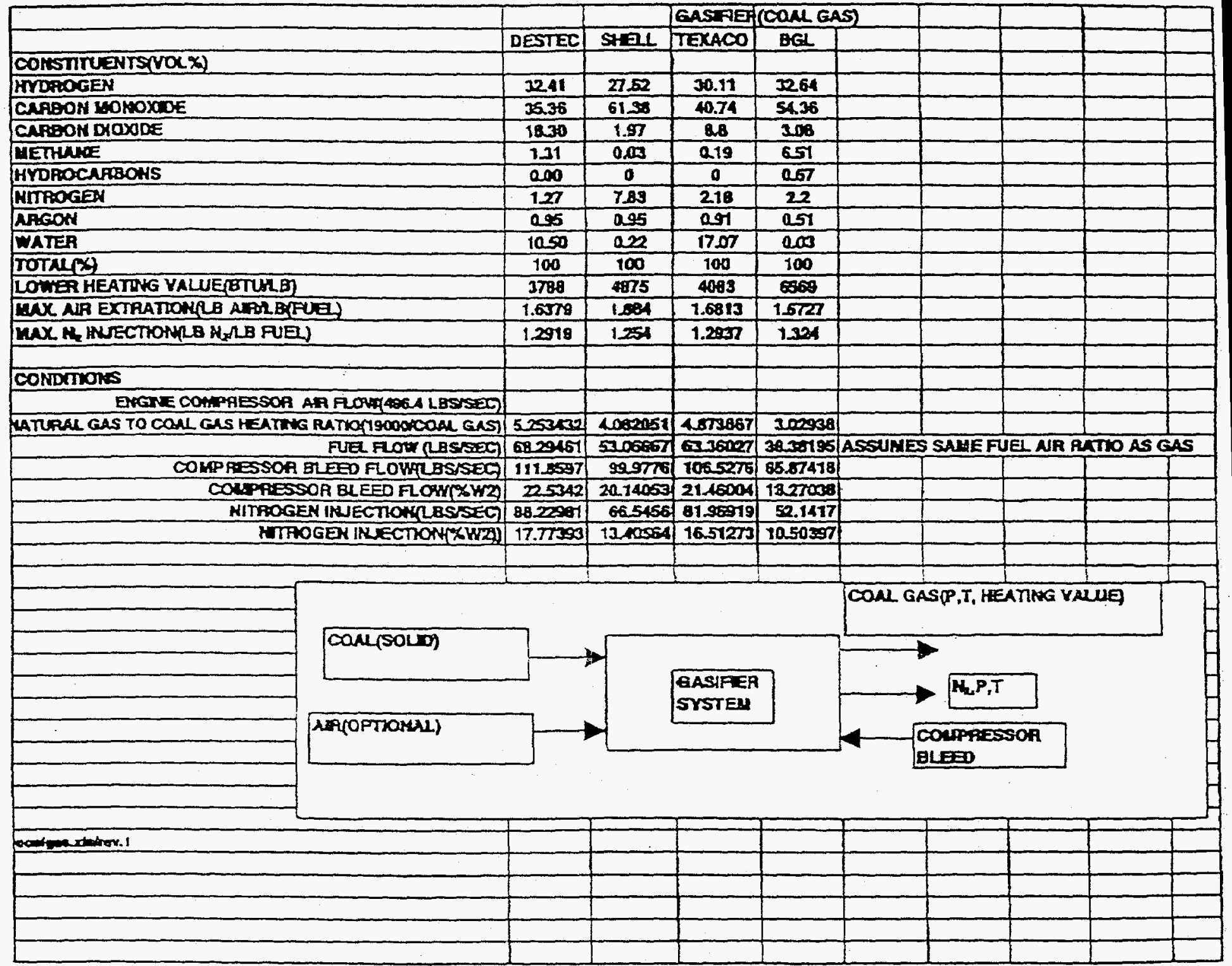




\section{TASK 4B - Utility CFATS}

\section{Background}

The GE STAG (steam and gas) 107H combined cycle has been under continuing evaluation for use in an Integrated Gasification Combined Cycle configuration. Particular emphasis has been placed on integration with the Shell-based gasification system which has been selected for a potential repowering application. This represents a special case for the STAG 107H system and involves a careful study of matching the combined cycle's steam bottoming cycle, the gas turbine's steam cooling requirements, and the need for operation of the IGCC design with natural gas startup and backup fuel. In addition to this site-specific activity, work has been accomplished on a general "greenfield" IGCC system design, again using the Shell-based gasification technology, but incorporating the full, single-shaft STAG $107 \mathrm{H}$ configuration.

\section{Progress During Reporting Period}

The "greenfield" or IGCC baseline configuration studies were run and demonstrated an ability to achieve over $400 \mathrm{MW}$ from a single IGCC flow train at ISO conditions. These initial estimates have been made without air separation unit (ASU) integration with the gas turbine's compressor, but with $\mathrm{N}_{2}$ injection, and have incorporated a larger first-stage turbine nozzle area design, similar to GE's practice with its " $F$ " technology IGCC configurations. Firing temperature has been limited by adherence to hot gas path operating temperatures consistent with projections for natural gas fired operation such that hot parts lives are unaffected by IGCC operation.

IGCC system requirements for the STAG $107 \mathrm{H}$ combined cycle have been identified and consist of the following categories:

- Increased gas turbine nozzle flow area

- Dual fuel, diffusion flame, gas turbine combustion system

- High-temperature gas turbine syngas fuel skid, piping, and turbine manifolds

- Nitrogen injection control skid, piping, and turbine manifolds

- Air extraction control skid, piping, and manifolds

- Syngas nitrogen purge system

- Fuel control system integration with gasification process

- Turbine and gas control skid modifications for high $\mathrm{H} 2$ syngas fuel

- Gas turbine cooling steam temperature control for both syngas and natural gas

- Thermal integration of syngas and natural gas fuel heating systems

- Optimization of high-pressure ASU with high-pressure-ratio gas turbine 


\section{Plans For Next Reporting Period}

- Further examination of $7 \mathrm{H}$ gas turbine compressor capabilities over a range of ambient air temperature operation with low Btu syngas fuel

- Emission performance estimates with syngas combustion in diffusion flame combustor designs being proposed for the $7 \mathrm{H}$ gas turbine

- Air extraction integration with the ASU. 


\title{
Task 8 - DESIGN AND TEST OF CRITICAL COMPONENTS
}

\author{
Task 8.1 - Particulates Flow Deposition
}

\section{Objective}

The primary objective of this task is to characterize the particulate generated in an operating gas turbine combined cycle (GTCC) power plant whose configuration approximates that proposed for an ATS power plant. In addition, the task is to evaluate the use of fullflow filtering to reduce the steam particulate loads. Before the start of this task, GE had already negotiated an agreement with the candidate power plant, piping and a filter unit had already been installed at the power plant site, and major elements of the data acquisition system had been purchased, all with GE funds. The remaining work involves completing assembly and checkout of the system, and then conducting measurements to meet the stated objectives. Using coolant extracted from an existing plant that most closely simulates projected ATS conditions, we will determine filtration- and operatingperformances for a candidate full-flow metal filter system operating at ATS entry conditions and characterize the coolant particulates into and out of the metal filter.

\section{Progress for This Quarter}

During this Quarter, the steam flow and filtration system was off-line while the centrifugal deposition rig was being installed in the power plant flow apparatus. However, the stainless steel filter cartridge has been removed, inspected, and reinstalled in its housing.

Contacts with GE Power Systems design engineers on system parameters have been continuing. A steam purity specification was prepared for the steam purity required for an ATS turbine to reach its 24000 hour Time Between Outages (for cleaning).

\section{Plans for Next Quarter}

The steam cartridge filter will continue to get exposure to steam flow to the centrifugal deposition rig in coming months. Its flowrate will only be approximately $1 / 3$ of earlier exposures because of flow specifications for the centrifugal deposition rig tip turn specimens, but the added exposure time will provide valuable material data and generate more opportunities for steam characterization.

\section{Task 8.1.1 - Coolant Purity}

\section{Objective}

The objective of this task is the exposure of typical ATS gas turbine airfoil cooling channel geometries to real steam flow to determine whether there are any unexpected deposit formations. The task is a static analog of the centrifugal deposition rig trials of Task 8.2, 
in which a bucket channel return bend is exposed to steam flow. Two cooling channel geometries are of primary interest in this static exposure.

The static specimens will be mounted in a coded pressure vessel (already available at the power plant from previous GE work) that would serve both to offer personnel protection because the specimens do not have standard ASME closure designs and to enable preheating of the specimens by flooding the vessel with steam as a heating medium before steam flow initiation.

\section{Progress for This Quarter}

During this Quarter, the flow specimens (based on prototype and current hardware) have been received and have been provided to the vendor to isolate the desired channels and to apply inlet and outlet ducts for each specimen. The pressure vessel, which will contain these specimens and provide a preheating steam environment, has been plumbed into place in the steam flow circuit.

\section{Plans for Next Quarter}

The current plan is to receive the flow specimens in January, flow check them, and mount them in the vessel. First exposure is planned to occur in February.

\section{Issues, Problems, Changes}

The centrifugal deposition rig must be commissioned prior to the static flow testing to be sure the static specimen flow loop doesn't influence the centrifugal deposition rig performance. It is expected that there will be no problems running the static specimens downstream in series with the centrifugal deposition rig.

\section{Task 8.2 - Particle Centrifugal Sedimentation}

\section{Objective}

The primary objective of this task is to determine the settling characteristics of particles in a cooling stream from an operating gas turbine combined cycle (GTCC) power plant when that stream is ducted through a passage experiencing the G-loads expected in a simulated bucket channel specimen representative of designs proposed for an ATS gas turbine. GE has identified a target power plant at which to site the experiment. Prior to the start of this task, GE had completed a proprietary computational code that was proposed to be useful in predicting particle trajectories. A motor-driven centrifugal deposition rig that accepts coolant at ATS conditions, accelerates it at conditions expected in specimens built to simulate ATS bucket coolant channels. Coolant flow conditioning and control is to be provided by the filtration and control system already utilized for the Task 8.1 work effort. Deposits collected in the specimen tip channels will be compared to predictions and to desired times between outages for the ATS when the channels could be cleaned. 


\section{Progress for This Quarter}

The equipment is installed at the powerplant, a punch list of items for completion has been issued, and a Pre-Operation Review has been held to examine the system. The Review consisted of eleven technical staff including engineers from the plant owner (1), plant operations supervision (3), GE Power Systems design and test engineers (3), centrifugal deposition rig vendor (2), and CRD staff (2). The Review identified over 20 items to review, the most important of which was to provide motor- and steam-supply-abort switches on the turbine level above the apparatus so that an operator would not have to be in the basement to trip the system. The computer system control program has also been completed.

\section{Plans for Next Quarter}

The plan is to finish the installation by putting in the computer system and starting commissioning trials in December. The centrifugal deposition rig will initially be bypassed to check out the control program and flush out the system. It will then be pressurized with steam.

\section{Issues}

The rotary coolant seals remain the largest unknown until the system is put into operation. Other issues being worked out include interim, non-destructive inspection of any internal specimen deposits with fiber optics and remote sampling probes. The largest uncertainty remains whether or not the actual steam leakage across the rotary face seal systems will be sufficiently low to enable full $4800 \mathrm{rpm}$ operation. If the rotor housing chamber is higher, then the rotation rate will have to be reduced (because of motor-power- and allowedspecimen-temperature limits) though the results will still be scaleable to ATS operating conditions even in that case.

\section{Task 8.2.2 - Rotor Materials Coolant Compatibility}

\section{Objective}

The objective of this task is to evaluate the effect of oxygen partial pressure on hightemperature crack propagation and crack tip oxidation in a material anticipated for structural application in ATS gas turbines. This evaluation will be accomplished by using hydrogen/water vapor/inert gas mixtures to control the equilibrium atmospheric oxygen partial pressure in the test chamber over a wide range of values. Compact tension specimens will be used to evaluate crack propagation properties.

\section{Progress for This Quarter}

Controlled-atmosphere fatigue crack propagation (FCP) testing began on 15 September 1995. 
Controlled-atmosphere, fatigue crack propagation (FCP) testing of compact tension specimens taken from a section of the GE Power Systems subscale forging began on 15 September 1995. Data from FCP tests have been collected for constant temperature, stress intensity range, and total pressure, as a function of cyclic period.

Sustained load crack propagation (SLCP) data have also been generated from a single compact tension specimen taken from a section of the GE Power Systems subscale forging. Samples were oriented such that the crack propagation plane was in the L-R orientation and crack propagation occurred in the radial direction. Testing was performed at constant temperature, stress intensity, and total pressure.

\section{Plans for Next Quarter}

Auger Electron Spectroscopy and Analytical Electron Microscopy will be used to investigate and compare the oxide scale compositions of samples tested in hydrogen and steam atmospheres.

\section{Task 8.3 - DETERMINATION OF DRIVING FORCES FOR THERMAL BARRIER COATING FAILURE}

Task 8.3.1 - TBC Testing and Analysis

\section{Objectives}

Development of thermal barrier coatings (TBCs) with improved life and reliability will require a comprehensive understanding of the mechanisms of degradation that occur during gas turbine service. There are multiple objectives in this task. One objective is development and confirmation of methods to measure and predict TBC stress states as a function of thermal and mechanical strains. This capability is fundamental to all quantitative TBC design and life prediction methodologies. An additional objective is development of a practical, versatile laboratory-scale thermal gradient exposure facility that is capable of simulating the extreme thermal conditions anticipated for TBCs in an advanced gas turbine. This atmospheric E-beam high gradient test facility will then be used to evaluate TBC-coated specimens in the thermal and stress states expected in service.

The specimen design ("tophat" specimen) for the E-beam thermal gradient test facility requires further analysis and modification to insure that it properly mimics the thermal and strain fields present in critical regions of a first-stage nozzle in an ATS gas turbine. Results from instrumented specimens will be used to compare observed versus predicted thermal and strain fields. Several geometric variables in the tophat design will be varied as necessary to confirm analyses and define improved specimen configurations.

Improved instrumentation and detectors will be developed for characterization of temperatures, fluxes and strains in specimens tested in the E-beam thermal gradient test facility. Types of instrumentation and detectors to be further developed include thin-film thermocouples, thin film strain gages, IR pyrometers, surface profile monitors and laser fluorescence of the thermally grown alumina layer. The measurement techniques must be 
compatible with the extremely harsh testing conditions and provide minimal interference with the intended temperature and thermal flux fields. The techniques will be used to further confirm validity of specimen designs and to provide monitors for feedback control during routine testing.

TBC durability will be evaluated in room temperature mechanical tests to complement thermally loaded specimens such as the E-beam fillet specimen described above.

\section{Progress for This Quarter}

\section{TBC Specimen Development and Testing}

A set of 5 validation tophats was machined to produce various stress levels at the bondcoat/TBC interface at temperature by control of the tophat face and wall thickness. After TBC coating, these samples are heavily instrumented (12 thermocouples and 8 strain gauges). These samples are tested at lower level, steady state conditions holding the backside metal temperature at a level to preserve the polymer substrate of the strain gauges. Validation testing maps the temperature and strain response of the sample to heat input. This map will then be used in ATS Phase 3 tasks to predict the sample strain state at the elevated ATS conditions from the thermocouple and sidewall strain gauges that remain at the higher temperatures.

Data from the set of 5 validation tophats have been reduced and sent to the numerical analysis group for analysis and comparison with modeling.

\section{Instrument Development}

The x-ray imaging system has been installed and used to monitor the $\mathrm{x}$-ray flux from the tophat face. This system will provide heat flux data to the numerical modeling group for incorporation into the model.

Laser fluorescence of the bond coat was measured (at room temperature). A peak was observed due to bond coat oxide. Some preliminary measurements were made at higher temperatures.

GE Aircraft Engines and other vendors have been approached in regards to high temperature thin film thermocouples and strain gauges. Little progress has been made in this portion of the task due to the severe operating conditions of the test.

Samples have been sent out for TBC optical properties measurement as a function of wavelength in order to allow proper choice of wavelength for pyrometric measurements of temperature and provide thermophysical data for radiation heat transfer calculations. Reflectivity data $(0.3-26 \mu \mathrm{m})$ will be obtained from porous and DV $\mu \mathrm{C}$ TBC coatings as a function of temperature $\left(25-1300^{\circ} \mathrm{C}\right)$. An advantage of reflectivity measurements is that sample preparation artifacts (such as polishing or thinning) are not required. The data will be used to calculate transmittance and emittance. Independent measurements of emittance at high temperature would also be useful, and will be looked into. 


\section{Fillet Region TBC Test}

The design of a tophat fillet specimen is complete. This specimen is designed to simulate the conditions predicted in the fillet region of the first-stage nozzle (the fillet between the airfoil and the endplate). A three-dimensional finite element model of the fillet specimen has been created in the ANSYS parametric design language. The specimen geometry and boundary conditions are specified via design variables. This approach allows sensitivity studies to be performed quickly. Target values for the fillet specimen geometry and temperature distribution were determined from the current first-stage nozzle design and numerical predictions for the cascade nozzle. Preliminary analyses of the fillet specimen have been performed to determine sets of dimensions and boundary conditions that achieve the target conditions. The backside heat transfer coefficient distribution has been varied to achieve a uniform temperature profile around the circumference of the fillet region.

Temperature and preliminary metal stress state targets were defined for the fillet specimen based on the anticipated operating conditions of the first-stage nozzle. Finite element analyses predict that these conditions can be achieved in the fillet specimen by varying the specimen geometry and boundary conditions. The E-beam heat flux profile and the backside coolant heat transfer distribution were found to be key parameters in achieving the desired stress state. Other combinations of temperature and stress states can be achieved by varying the experimental conditions and/or the specimen geometry. The assumed boundary conditions, in particular the E-beam heat flux and coolant heat transfer coefficient distributions, were verified using data from validation tests of heavily instrumented specimens. The preliminary design of the specimen is now complete, and 16 castings have been ordered from the vendor. The proposed experimental conditions will be updated after the values of the stress targets have been finalized.

Six stainless steel tophats have been fabricated to use as APS spray test pieces, in order to develop the robot motions to deposit TBC on the fillet tophats which are representative of those found on the ATS machine in that location.

\section{Plans for Next Quarter}

The data obtained from the validation testing will continue to be compared to the results of the numerical model of the tophat.

Thin film thermocouples and strain gauge thermocouple discussions with GE Aircraft Engines and other vendors will continue. Other instrumentation will be installed (laser surface profilometer). Laser fluorescence of the bond coat will be measured at higher temperature.

Design of the mechanical test samples will be continued.

APS parameters for the deposition of TBC onto the fillet tophats will be developed. 


\section{Task 8.3.2 - LCF Life and Crack Propagation}

\section{Objective}

The objective of this task is to evaluate the low cycle fatigue (LCF) life of the most critical regions of the ATS turbine inlet nozzle using representative geometries, thermal fields, and stress/strain fields. Testing will be performed under various conditions, with standard defined cycles and with accelerated cycles. Specimens will be tested as practicable to predicted life cycles, then accelerated until failure. Failure may be either TBC loss or crack-through of the metal substrate. Some specimens will continue to be tested after crack-through to determine the crack propagation rate, i.e., rate of leakage increase. All specimen data will be used in the validation and updating of life prediction models for $\mathrm{TBC} /$ metal systems.

\section{Progress for This Quarter}

One purpose of this task is to evaluate the LCF life of critical regions of the ATS Turbine Inlet Nozzle. High thermal gradient E-beam testing is being used to expose superalloyTBC specimens to temperature and stress levels both representative of the ATS turbine inlet nozzle and far in excess of expected conditions, to determine LCF life of the metal within reasonable test durations. Current efforts are focused on stress/life analyses and testing in support of the turbine nozzle development.

\section{Testing}

Electron beam rig modifications were completed to achieve higher specimen temperatures while maintaining elevated metal-TBC interfacial stress levels. The insulating cover of zirconia felt was replaced with a water-cooled copper plate having a center aperture for passing the required E-beam energy flux. The helium cooling jet array geometry was modified to direct more coolant to the tophat side walls.

Following modifications, a new fully instrumented, plain tophat specimen was installed in the rig.

This first LCF tophat specimen, known as LCF \#1, completed the first period of testing without substrate failure, at which time the specimen was removed for non-destructive evaluation (NDE). This specimen has porous, Class C TBC. The specimen was replaced in the test rig and cyclic testing continued.

A duplicate specimen has been tested to failure in the E-beam facility. This specimen is known as LCF \#2.

Non-Destructive Evaluation and Metallography

NDE performed on LCF \#1 included eddy current inspection, thermal wave imaging, profilometry, and photography.

Specimen LCF \#2 has undergone ultrasound inspection. 
This specimen has been sectioned to allow the fracture of the cracked region for fractography analysis to determine, if possible, the number of cycles to crack initiation.

\section{Stress and Life Analyses}

During the course of initial LCF testing, analyses were performed by CRD and GE Power Systems engineers in concert to determine a set of new test conditions required to obtain reasonably short test times while maintaining metal stress levels within the range of available isothermal LCF data.

Post-test analyses have been performed using the experimental data from LCF \#1 specimen to validate temperature and strain predictions. Analyses were carried out at two low temperature conditions. In both cases, the comparison between the finite element model thermal prediction and the thermocouple readings from the interior of the tophat is very good, lending confidence to the thermal boundary conditions used in analysis.

Cyclic creep calculations have been started to estimate the effects of bond coat and substrate relaxation on the stress state after specimen cool-down. The residual stresses may provide insight into the observed cracking of the bond coat and metal after E-beam cycling, as well as the delamination of the TBC.

\section{Other Activity}

Two additional plain tophat specimens have been instrumented for LCF testing. The first of these specimens was installed and testing begun. No appreciable test time had been accumulated on the specimen.

Six additional plain tophat specimens have been machined to dimensions similar to those of the currently running tophat. These specimens will be coated and instrumented for use in obtaining a statistically significant set of data for LCF life with high thermal gradient.

Experience with the E-beam facility is allowing for longer total test times per day of operation. Efforts are continuing toward near 24-hour operation such that elapsed specimen test times will be reduced.

\section{Plans for Next Quarter}

LCF testing will continue during the next quarterly period, with LCF \#3 and \#4. Post-test evaluations and analyses will also continue.

\section{Task 8.3.3 - Bond Coats for Improved TBC Thermal Cycle Life}

\section{Objective}

Recent experiments with alternate bond coat application techniques and alternate bond coat chemistries indicate that significant improvements in the thermal cycle life of a TBC system can be achieved through bond coat modification. An experimental matrix containing a bond coat chemistry other than the one proposed for use in the ATS TBC system 
will be established to assess the role of optimized bond coat processing on TBC thermal cycle life.

Bond coat process parameters and post-heat treatment sequences will be selected to alter bond coat mechanical properties. All bond coats will be coated with an approximately 0.038 -cm-thick (0.015-inch-thick), dense vertically microcracked, air plasma sprayed (APS) TBC and evaluated in thermal exposure. Compressive shear resistance and other mechanical properties of the various TBC systems will be evaluated as a function of exposure time, exposure frequency, and exposure temperature in cyclic oxidation tests. The influence of bond coat properties on TBC life will be determined for the new system.

\section{Progress for This Quarter}

\section{Experimental Matrix and Choice of Bond Coat Parameters}

A test specimen matrix was established which emphasizes materials and processes critical to strength, mechanical properties and durability of ATS coatings.

The thermal exposure cycle is designated as a furnace exposure test (FET) which utilizes a furnace heatup from room temperature to a 10 -hour hold at the selected temperature, followed by a furnace power-off cooldown. Four furnace cycle tests are being conducted for each temperature. The times have been selected such that groups of specimens can be extracted at increasing exposure time and, consequently, varying degrees of thermal distress. The fourth time group is being cycled to failure. FETs are being cycled only to failure without extractions at intermediate times.

Sample groups extracted prior to failure are being evaluated metallographically to determines oxide growth rates and to determine length, location and nature of propagating cracks. The scanning electron microscope will be used to evaluate microstructural changes in fracture surfaces of the TBC as a function of exposure time Mechanical properties of the coatings at various stages of thermal cycling will be determined using tests for tensile pull strength, compressive shear strength of the attached coating, and Young's modulus of the detached coating. Arrhenius plots are being constructed from the failure time data and will be used as a guide in defining failure mechanisms.

\section{Furnace Exposure Testing and Furnace Cycle Testing of Specimen Matrix}

During this reporting period, enough specimens have come off furnace cycling tests to permit the plotting of predictive data.

The test matrix covers temperature trials and two different thermal cycling rates at each temperature. Coating strength and mechanical properties will be measured as a function of cycle time and temperature.

\section{Plans for the Next Reporting Period}

\section{Furnace Cycle Testing of Specimen Matrix}

Furnace cycle testing of specimens will continue. The furnace cycle test (FCT) heats the furnace rapidly from room temperature to a 45 -minute hold at the selected temperature, 
followed by a forced air quench on each return to room temperature. Metallographic examination of failed specimens and specimens withdrawn prior to failure and will continue, and mechanical testing of coated specimens will begin.

\section{Task 8.3.4 - Critical TBC Properties - Thermal Conductivity}

\section{Objective}

Accurate measurements of TBC thermal conductivity are important for thermal analysis and design of coated turbine components. Current practice uses conductivities that are measured with laser flash techniques that directly evaluate the thermal diffusivity. In this task, methodology and apparatus will be developed for measuring TBC thermal conductivity by an independent means. The effective thermal conductivity of various TBC systems including TBC, bond coat, and metal substrate will be determined by a transient technique employing convective heat flux. Instrumented test plates will be heated to a high temperature and subsequently cooled with large-diameter, air impingement jets at room temperature to provide a uniform heat transfer coefficient over the test article of interest. Instrumentation data and known heat flux will be used with finite element modeling to determine the effective thermal conductivity of the system and the TBC thermal conductivity.

\section{Progress for This Quarter}

In order to determine the thermal loading on the first-stage nozzle and other TBC-coated components of the advanced machine, an accurate determination of the TBC thermal conductivity must be provided. There are many issues involved in making accurate TBC thermal conductivity measurements including accurately characterizing the boundary conditions of the test specimen, the TBC surface temperature, base metal and TBC bulk properties, and the advantages and disadvantages of using a transient vs. a steady-state test.

To help resolve these issues, a transient conduction computer model has been generated to help design the TBC thermal conductivity experiments and rig hardware. One potential stumbling block will be characterizing the radiative cooling on the test specimen due to difficulties in accurately determining TBC surface temperature and spectrally-averaged emissivity. To circumvent this problem, a transient thermal resistance matching experiment has been designed which does not require accurately determining the TBC-side boundary condition but, instead, only requires that it is repeatable from test to test. The basis of this experiment is measuring the transient response of the backside surface temperature of a heated specimen subject to convective and radiative cooling on the opposite surface. The TBC thermal conductivity can be determined by comparing the backside surface temperature response of the TBC-coated specimen with that of uncoated specimens of different thickness (i.e., different thermal resistance) until a good match is achieved. This test rig is simpler than previous designs as it requires one heat source (natural gas flame), fewer thermocouples, and easily-obtainable low-conductivity insulation. Also, thermal modeling of the system is made easier by providing an insulation boundary condition on all sides except the TBC-coated surface. 
In the steady-state test, a known thermal gradient across the test specimen will be applied by independent natural gas flames. The base metal of each specimen will be instrumented with thermocouples in order to determine the heat flux through the metal and, consequently, through the TBC. In order to directly determine the TBC thermal conductivity, the surface temperature of the TBC must be determined. Currently, an infrared pyrometer is being investigated to serve this purpose. Further analysis is needed, however, before the feasibility of accurate TBC surface temperature measurements with this tool can be established.

\section{Plans for Next Quarter}

The experimental program for TBC thermal conductivity (diffusivity) will be completed. Several specimens with different TBC characteristics will be tested in order to accurately quantify the TBC characteristics over a wide range of conditions.

\section{Task 8.4.1 - Advanced Machine Hot Gas Path Seals}

\section{Objective}

The objective of this task is to design, fabricate, and install a high temperature static seal test rig capable of testing advanced seal concepts at $537^{\circ} \mathrm{C}\left(1000^{\circ} \mathrm{F}\right)$ and $2.412 \mathrm{MPa}$ (350 psi), providing $0.45 \mathrm{~kg} / \mathrm{sec}(1 \mathrm{lb} / \mathrm{sec}$ ) of air flow. The rig will be checked out for proper operation using some of the seals being developed for the ATS gas turbine.

\section{Progress for This Quarter}

Junctions between turbine stator segments have to be sealed in order to maintain high efficiency. Such junctions, typically, have slots on mating edges. Seals are used in the slots, bridging adjacent members, to block off any leakage. The hot gas/air in these locations sees high pressures ( $350 \mathrm{psi}$ in some locations), temperatures ( $1000^{\circ} \mathrm{F}$ or higher) and pressure-differences of over 150 psi in some sections.

A high temperature seal test rig has been designed to ASME pressure vessel code. This rig will handle pressures of up to $2.412 \mathrm{MPa}(350 \mathrm{psi})$ and temperatures of about $537^{\circ} \mathrm{C}$ $(1000 \mathrm{~F})$. The hot air requirement is a maximum of $0.4535 \mathrm{~kg} / \mathrm{sec}(1 \mathrm{lb} / \mathrm{sec})$. The basic design of the high temperature rig comprises a pressure vessel with a "diaphragm" dividing it into a high pressure chamber and a low pressure one. The diaphragm has a hole on which is bolted a jaw. The rig is a pressure vessel divided into a high-pressure chamber and a low-pressure chamber by a diaphragm. A jaw unit is bolted on the hole. This jaw unit comprises a pair of jaws with slots, each of which is $30.5 \mathrm{~cm}$ (12 in) long. The rig is designed to accept a variety of jaw units with a selection of slots. Tests are done using jaws with slot geometries appropriate to the ATS gas turbine; the results will be used to finalize the definition of the slot geometries. Mismatch, offset, and gap conditions can be varied by means of the jaw unit assembly. 


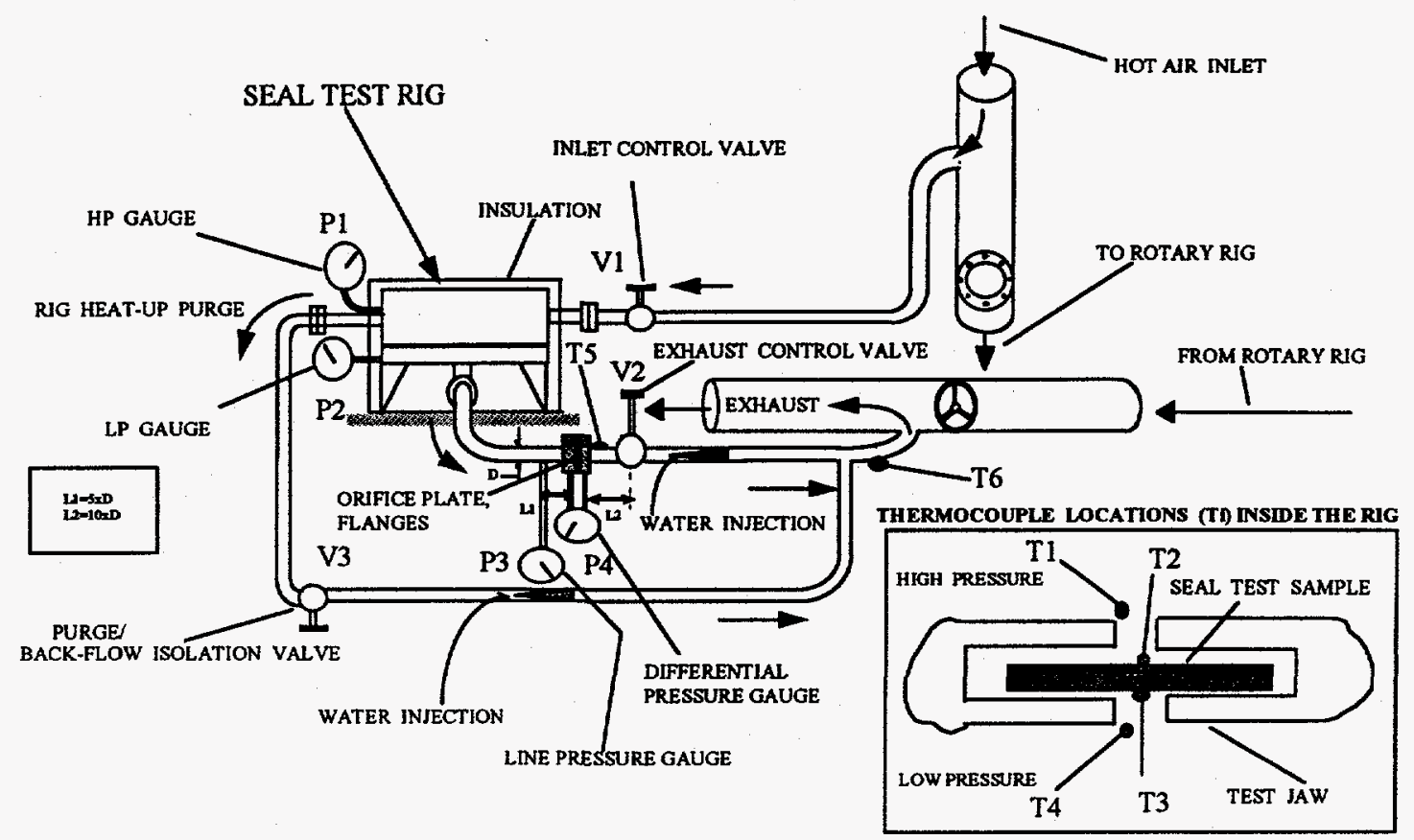

Figure 8.4.1-1. High Temperature Seal Test Rig System Diagram

The following key items of progress are shown in Figure 8.4.1-1.

1. The high temperature rig has been fabricated, and hydrotesting is underway.

2. All high temperature valves (V1, V2, and V3) have been procured; extension handles for these have been fabricated.

3. All pressure taps (transducers: P1, P2, P3, and P4) and corresponding digital pressure gauges have been procured.

4. All thermocouple wires and lead-in tubing ( $\mathrm{T} 1$ to $\mathrm{T} 6)$ have been procured.

5. Seal test jaw-holder fabrication is in progress; expected to be completed FW 51.

6. Seal jaws fabrication is in progress; expected to be completed FW 51.

7. Thermal insulation unit for the rig has been fabricated.

8. Rig installation table has been delivered.

9. Facilities work (piping and site preparation, insulation) is in progress; expected to be completed by $96 \mathrm{FW} 03$.

10. Sealing rope and other accessories are on order.

11. The high temperature rig installation system layout has been designed and facilities work to install plumbing, etc., is underway. 


\section{Plans for Next Quarter}

The installation of the high temperature rig will be completed. The Standard Operating Procedure will be reviewed. A Pre-Operation Review will be held and check-out tests will be performed.

\section{Task 8.5 - Enhanced Impingement Heat Transfer}

\section{Objective}

Tests will be performed to evaluate a new concept for backside impingement cooling aimed at lessening the adverse effect of cross-flow on air jet thermal dilution and to determine the upper limits for jet heat transfer at higher air supply pressures.

For long impingement channels, the spent jet impingement air represented as cross-flow mixes and raises the temperature of the cold air jet before it strikes the hot wall.

\section{Progress Since Last Reporting Period}

This task has been completed.

\section{Plans for Next Reporting Period}

This task has been completed.

\section{Task 8.6.1 - Rotational Heat Transfer - Bucket Cooling}

\section{Objective}

Prediction of gas turbine blade life requires sufficient accuracy in the prediction of both the local hot gas side and coolant side heat transfer coefficients present at the relevant blade surfaces. While a considerable data base exists for the hot gas side coefficients, the data base for the rotating blade coolant passages is very limited. Only recently have measurements in rotating simulated blade passages become available that cover the conditions of interest to aircraft gas turbine blades. At the conditions present in the ATS turbine, extrapolation of the existing data base is needed. An effort is presently underway at CRD that will provide the required heat transfer data base over the range of dimensionless parameters of interest to the ATS turbine.

\section{Plans for Next Quarter}

Data acquisition up to maximum Buoyancy numbers for the increased aspect ratio test duct in the full scale test rig will be resumed. These data should be completed by the end of February 1996. 


\section{Task 8.6.2 - Rotational Heat Transfer - Wheelspace Cooling}

\section{Objective}

The interstage turbine wheelspace, diaphragm, and outer seal design, as well as the operating conditions for the ATS turbine, are sufficiently different from existing gas turbine experience to render the use of normal purge flow criteria questionable for preventing hot gas ingestion and for providing sufficient cooling of this region. CFD computations have been used to select a design concept that will be verified in the proposed experiments. An existing GE Freon gas rotational test facility will be modified to simulate the ATS turbine wheelspace geometry. The Rotational and Radial Flow Reynolds numbers present in the ATS gas turbine design can be matched using a 3:1 reduced scale model operating with R$134 \mathrm{~A}$ at about 21 psia in the cavity. Leakage across the nozzle stage and the effect of the circumferential pressure variation on ingestion will be measured. This testing will allow rapid evaluation of various design approaches.

\section{Progress for This Quarter}

Test rig construction has proceeded during the last quarter with completion of shop work scheduled for mid December 1995. The test rig assembly is shown in Figure 8.6.2-1 in its major parts together with an enlarged view of the rotor-stator seal configuration. The dimension shown in Figure 8.6.2-1 is in inches. CFD computations of the seal behavior have been carried out over a range of cooling air flow rates both with and without circumferential pressure gradients. The CFD predictions will be verified subsequently by the data to be obtained with the present seal configuration to allow the evaluation of other seal configurations or cooling air flow splits with a validated CFD model. 


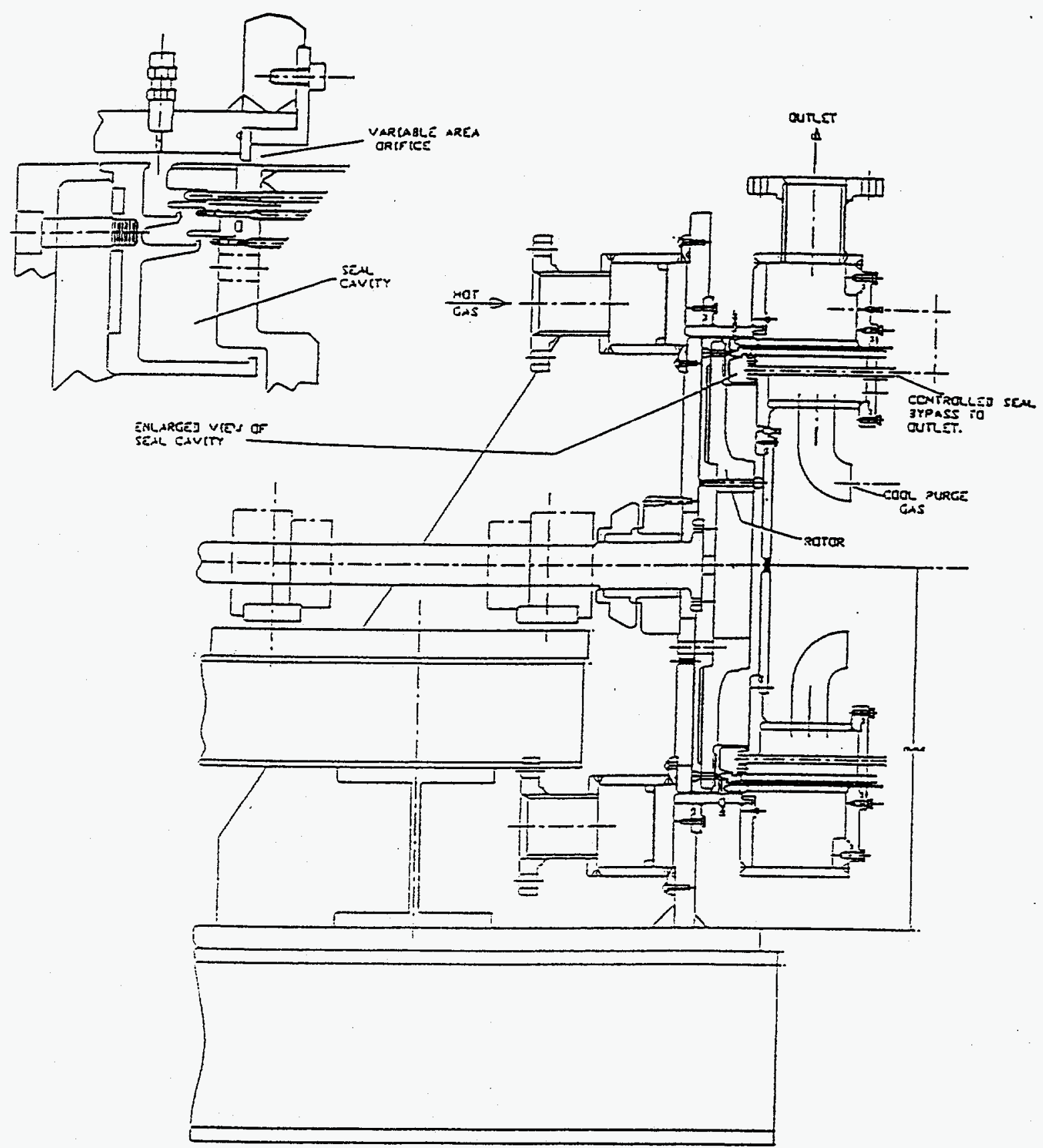

Figure 8.6.2-1. Wheelspace Cooling Test Rig Configuration. (Dimensions are in inches.)

Since the circumferential pressure gradients will be imposed by a variable area orifice downstream from the seal location, see Figure 8.6.2-1, the CFD code was used to calculate the orifice geometry needed to establish a 3-4\% circumferential pressure gradient over about 11 equal sectors. The latter is over three times larger than the anticipated gradients with the circumferential spacing defined by engine design characteristics that may cause such gradients. When no gradients were imposed no hot gas ingestion was calculated even at significantly reduced cooling air flow. When a $3 \%$ circumferential 
gradient was imposed significant hot gas penetration through the outer seal was calculated at a nominal design cooling air flow.

\section{Plans for Next Reporting Period}

The test rig construction and installation is scheduled for completion by the end of February 1996. This includes instrumentation and test facility modification needed to accommodate the test rig.

\section{Task 8.7.6 - Combustion Instability}

\section{Objective}

The objectives of this task are (1) to understand the physical mechanisms responsible for the onset and sustenance of dynamics in lean premixed combustion systems, (2) to obtain detailed experimental data (consisting of frequency, amplitude, rms level, mode shape, and correlations) in the combustor for various upstream and downstream boundary conditions and various fuel delivery system boundary conditions; and (3) to enhance computational capabilities in the form of improved sub-models to better quantify upstream and downstream acoustic boundary conditions and fuel delivery system impedances and their effects on dynamics.

\section{Progress for This Quarter}

1. The task comprising two-burner tests to simulate the possible coupling of two combustors on a gas turbine via the gap between the transition piece and first-stage nozzle is complete.

2. In the task related to understanding the dynamic response of the air and fuel systems, bench testing of the special high-speed rotating-valve has been completed. This valve will provide controlled periodic pressure oscillations within the combustion chamber under cold flow to simulate the oscillations observed during combustion dynamics. Since the oscillations are forced and can be run without the added complexities of a combustion test, the valve will provide better access to detailed measurements. The bench tests confirmed that strong pressure oscillation strength could be generated with the valve, particularly when matched to the resonant frequency of the test rig. Oscillation strengths of $10 \%$ of the mean pressure were demonstrated with the bench system in agreement with predictions. Installation and testing in the actual test rig is proceeding.

An instrumented fuel nozzle has been built to support dynamic pressure measurements within the fuel passage. The nozzle is based upon a production low-NOx combustor nozzle. A phase-resolved gas sampling system was developed previously and is ready for check-out once rig testing starts.

3. Progress has been made on the sub-model used to calculate the impedance of the fuel delivery system. Several additional features have been added to the acoustic circuit model of the fuel passage to allow the internal pressure oscillations to be calculated for comparison to data. 


\section{Plans for Next Quarter}

The rotating valve will be installed in the test rig and tested for operability and oscillation strength with actual air flow and production hardware. Dynamic pressure in the combustion liner will be measured, and the excitation frequency will be varied to map the resonant frequencies of the rig. Once a reasonable oscillation strength is demonstrated in the real test rig, measurements of the dynamic response of the fuel and air systems will then be taken with controlled oscillations using the rotating valve system, and the results will be used to validate dynamic models.

The CFD model will be applied next to experimental combustors to obtain direct validation using existing data. Effects of reaction rate and boundary conditions will be evaluated versus experimental conditions. The sub-model for the fuel system response will be compared with data from the rotating valve test described above.

\section{Task 8.7.7 - Fuel Heating}

\section{Objective}

Heated fuel can provide improved performance for the ATS machine but must be investigated experimentally to evaluate the effects of hot fuel on system performance and combustion dynamics. One of the technical concerns in the use of fuel heating is carbon buildup in the fuel passages between the heat exchanger and the fuel injector. In this task, a series of exposure tests, using natural gas with various impurity loadings, will be run for conditions representing the operating range of the ATS gas turbine. In addition, techniques to coat large in-situ piping with coke barrier coatings (CBCs) will be developed and evaluated.

Systems issues that affect overall performance will also be considered. An analysis of the fuel delivery system, including the fuel injectors, will be performed to determine the sensitivity to parameters such as pressure drop that influence combustion dynamics. A fuel control strategy will then be developed to address these systems issues.

\section{Progress for This Quarter}

Testing this quarter focused on the potential for carbon deposits to form in heat exchanger fuel passages during fuel heating.

A facility for high pressure testing was assembled. This facility features a boost compressor for natural gas and interlocked solenoid valves for unattended operation.

\section{Plans for Next Quarter}

Work towards objectives is proceeding well and will continue in the next period. The focus of activity will be continued high pressure testing, continued coating development work, and systems analysis of the heat fuel system. 


\section{Task 8.7.8 - Turbulence Intensity Probes}

\section{Objective}

A turbulent heat transfer probe has been designed to allow measurement of free stream turbulence in a DLN combustion system under full fired operation. This effort will provide for testing of this probe in a combustion environment using one or more of CRD's combustor rigs. This task will also provide testing support in available GE Power Systems combustor test stands, including the ATS Full Scale Nozzle Cascade Test Stand.

The ATS Turbine Inlet Nozzle Cascade will be improved by replacing the aluminum flowpath frame with a stainless steel frame. Other modifications will be made to improve the durability and interchangeable nature of the instrumented airfoils.

\section{Progress for This Quarter}

Testing of thin-film micro-sensor probes in combustion fed environments is sought in this task for the purpose of evaluating the ATS turbine inlet nozzle freestream turbulence intensity level, which has a major and direct bearing on the heat load for the nozzle airfoil and endwall. Upgrade modifications to the ATS turbine inlet nozzle cascade for the purpose of increased durability and flexibility is included in this task.

One of the recently received probes of the original design (dynamic design) has been sent back to the manufacturer for use in comparative calibrations of two new probes (static design). Each probe will be exposed to a high energy jet flame while being internally cooled, to develop a high sensor heat flux..

Progress on the ATS Turbine Inlet Nozzle Cascade this period includes (1) ordering of a machine base, rails, and pillow blocks for better cascade movement and change access, (2) heat treatment of the forged block of stainless steel prior to start of machining for the new cascade frame, (3) completion of the EDM wire-cutting effort, inside and outside, and (4) initiation of $\mathrm{CNC}$ machining to final dimensions and features.

\section{Plans for Next Quarter}

During the next quarter, both dynamic-mode and static-mode turbulence intensity probes will be tested in the Full Scale Nozzle Cascade and CRD's Catalytic Combustion Rig. The new ATS Turbine Inlet Nozzle Cascade frame will be installed, along with the associated support system.

\section{Task 8.7.9 - Turbulent Heat Transfer - Static Components}

\section{Objective}

The objective of this task is to obtain the necessary heat transfer boundary conditions to predict the metal temperatures and gradients of the first-stage nozzle airfoils, as influenced by the cooling mechanisms used in the various portions of the nozzle airfoil. Although highly effective in keeping the airfoil cool, the innovative cooling approaches used in the nozzle may impose 
high thermal gradients through the airfoil walls, making accurate knowledge of and optimization of such cooling critical. Internal convective heat transfer distributions will be supplied by two experimental models, one determining the impingement heat transfer characteristics within the several internal airfoil cavities, the other determining the specific impingement heat transfer enhancements due to the addition of various internal surface features such as miniature pin-fins. Both experimental models will utilize the thermochromic liquid crystal technique.

An investigation will also be made of the pressure drop and heat transfer coefficient distributions for first-stage nozzle airfoil spanwise impingement passages, and the results obtained will be compared with the values expected from the design methods.

The nozzle trailing edge air film cooling will be evaluated with warm rig testing to determine the most effective geometry for minimal coolant usage.

\section{Progress for This Quarter}

\section{Surface Enhanced Internal Heat Transfer}

The test section used for the impingement heat transfer tests is in a high pressure enclosure that can be operated at pressures of up to $10.2 \mathrm{~atm}(150 \mathrm{psia})$ by means of a back pressure control valve The impingement air is fed to a supply chamber. The air supply pressure and temperature, as well as the flowrate, are measured by means of calibrated pressure transducers. The test rig is equipped with square impingement jet plates $(5.08 \mathrm{~cm} \times 5.08 \mathrm{~cm})$ that can accommodate several jet hole configurations. The impingement test surface $(5.08 \mathrm{~cm} \times 5.08 \mathrm{~cm})$ is in intimate contact with a copper block that is heated by three cartridge heaters. The power dissipated by the heaters is measured by a wattmeter. The impingement test plates, positioned at a controlled distance from the impingement jet plates, are instrumented with four imbedded thermocouples that measure the plate temperature on the side facing the heater block. Tests are conducted at various jet Reynolds numbers ranging from $1 \times 10^{4}$ to $4 \times 10^{4}$.

\section{Test Procedure}

The test procedure involves setting up the impingement jet flow rates and then increasing the power in the cartridge heaters, to raise the impingement wall temperature to any desired temperature (maximum of $93.3^{\circ} \mathrm{C}\left(200^{\circ} \mathrm{F}\right)$ ). The air flow rate, the power dissipated by the heaters and the impingement plate temperatures are continuously monitored by a computer controlled data acquisition system. For a given flow rate, once a steady state is achieved, all the relevant parameters are recorded for analysis.

\section{Flow Tests with Manufactured Impingement Inserts}

In preparation for the high temperature first-stage nozzle cascade tests, impingement inserts for the side walls and the airfoil are being manufactured by GEPG. Flow tests are being conducted with the various inserts and the static pressure distributions are being measured for each insert in a fashion similar to the one described above under "Flow 
Checks of Impingement Insert." The results obtained with the impingement insert agree with the data discussed above and obtained with the test impingement insert.

\section{Trailing Edge Film Cooling}

The two objectives for this film testing are:

1. Determining the most advantageous film cooling geometry for use on the turbine inlet nozzle trailing edge region, where air film cooling will be employed.

2. Measuring the adiabatic film effectiveness downstream of the film injection under conditions representative of the mainstream flow field, the coolant-to-gas density ratio, and the blowing ratio.

Two tests have been set up to model the pressure side and suction side film injection locations, each with the ability to change film injection plates to test desired geometries. Following is a summary of pressure side film results, including additional information on flow conditions and geometries.

\section{Pressure Side Model Test Section Conditions}

The test was set up in our Warm Wind Tunnel. The tunnel configuration is simply a constant cross section of $7.62 \mathrm{~cm}$ by $10.16 \mathrm{~cm}$ (3 in by $4 \mathrm{in}$ ), with film cooling introduced through removable test plates in one wall of the tunnel. The wind tunnel was modified for these pressure side tests by replacing the opposing wall to the film injection plate with a converging wall.

While the insensitivity of the narrow slot film to blowing ratio is a desirable feature for design, the actual performance of the slot film is far below any CFD predictions. As is seen with the deep chamfer, the mixing of the coolant and the gas is critical to film performance. The mixing of coolant and gas, as well as associated influences such as freestream turbulence intensity, must be captured by CFD before CFD predictions can match experimental results.

\section{Plans for Next Quarter}

\section{Surface Enhanced Internal Heat Transfer}

Tests will continue with several other jet plates and test plate 10 to complete the data matrix for correlation development.

Nozzle Airfoil Impingement

Data analysis will be completed and a report will be drafted.

\section{Trailing Edge Film Cooling}

Suction side film testing will be completed. 


\section{Task 8.7.10 - Transition Piece Design Tools}

\section{Objectives}

Higher firing temperatures in the ATS gas turbine make the transition piece a crucial component. Flow separation in the transition piece must be avoided since it results in locally high heat transfer that could lead to liner burnout. Improved analytical prediction of flow separation in transition pieces will reduce the required amount of testing and reduce design risk. Faster meshing capability will allow for increased use of CFD analyses on complex transition piece geometries, reducing cycle time. One objective of this project is the further development of a 3D unstructured meshing system to make it more automatic and robust for complex transition duct geometries. This development is being carried out through a subcontract with MIT. Another objective of the project is to improve the ability to predict flow separation in transition pieces through the use of adaptive meshing and advanced turbulence models.

\section{Progress Since the Last Reporting Period}

\section{MIT Subcontract}

MIT has modified the structure of the geometry modeler within the surface mesh generator so that the task of reading PARASOLIDS models becomes straightforward. The structure of the new code should be able to support PARASOLIDS and FELISA geometries as well as a combination of both.

Work has started on assembling a graphical output interface. In the initial stages, this interface will allow for the visualization of the geometries and the meshes generated. Future developments include the possibility of interactively defining the background grid characteristics and eventually performing geometry "repair" operations.

The surface triangulation has been modified to allow for arbitrary stretching of the patches defining the geometry. Work is in progress to allow for periodic surfaces as well as floating (wake) surfaces. The mesh generator is now coupled with the Geometry Viewer. The Geometry Viewer has passed through the first phase of functionality. Current effort is being spent in being able to display Parasolids geometry.

MIT has completed the implementation of a directional refinement module. This module allows for the generation of grids that are stretched in one direction (e.g., for leading and trailing edges resolution) and two directions (e.g., for boundary layers). The code is being tested and has already produced the first results. Unlike the stretching obtained using a linear transformation, the algorithm used produces elements with the largest angles being close to $90^{\circ}$. The code can already handle floating surfaces (such as wakes) and different volume regions. Periodic surfaces are also allowed.

\section{Improved STAR-CD Meshes}

Several improvements are being made to the meshing script to pick up areas of flow separation. The turbulence modeling has been changed from the standard $k-\varepsilon$ formulation 
to a modified k- $\varepsilon$ formulation based on Renormalization-Group (RNG) theory. This will improve accuracy when modeling separated flows. Unfortunately this change, by itself, is not enough to predict separation in all regions of the model. Improvements in the differencing scheme have been investigated. A great deal of work was done to determine the optimal blend of upwind differencing and central differencing. The central differencing scheme is more accurate because it is less diffuse, but it is also very slow to converge. It was hoped that if a blend of upwind and central differencing was used, the model would be able to pick up flow separations more readily and still converge monotonically. The work done this quarter suggests that the "optimal" blend is to use pure central differencing, if possible. When enough central differencing is blended into the differencing scheme to pick up known areas of separation, the convergence is no better than when pure central differencing is used. Fortunately, the model does converge when a pure central differencing scheme is used; it is just slower and less monotonic.

Progress was also made in the user interface. It is no longer necessary for the user to pick the inlet and outlet planes while the script is running; however, user input has not been completely eliminated, and work will continue in this area.

\section{Plans for Next Quarter}

\section{MIT Subcontract}

MIT is making very good progress. As soon as the link with PARASOLIDS is fully functional, we will get a very powerful tool. We have provided MIT with some PARASOLIDS models to use to test the new software. We expect to get the first prototypes of the software in late December or early January. A visit to MIT is planned at that time to review the status of the software and see a demonstration of the prototype versions. When we receive the prototype codes, we will attempt to mesh and solve a typical transition duct geometry with the system.

Some effort is needed to account for cases where the surface grid needs to be refined due to the boundary recovery process in the 3D generation. This will be a focus of MIT's effort in the next month.

The next stage in the development of the graphical interface will be to incorporate a capability for "picking" and "selecting" geometrical entities. In this way, background meshes can be created interactively if needed. In addition this capability will allow for the interactive definition of wakes and other surfaces.

\section{Improved STAR-CD Meshes}

Efforts will continue to improve both the accuracy of the STAR-CD calculations and the automation of the meshing script. The following changes and improvements will be made. The mesh in the boundary layer will be increased to allow a profile shape to develop. Improvements will be made to the modeling of the boundary conditions at the exit of the transition piece. Currently the exit is modeled as a constant pressure boundary occurring at the physical end of the transition piece. It has been suggested that moving the constant pressure boundary away from the physical end of the transition piece improves the ability of the code to predict separation; this will be further investigated. 


\section{Task 8.7.11 - Effects of High Mach Number Diffuser}

\section{Objectives}

Proposed compressor exit designs for the ATS gas turbine give much higher exit Mach numbers than have previously been experienced. Potential difficulties include high combustion dynamics, a need for higher combustor system pressure losses to cool hardware, and flow field imbalances that increase the difficulty of obtaining low emissions. Studies are required to quantify risk and evaluate alternate designs. At CRD, flow tests on existing multi-combustor models will be carried out for validation via flow visualization and pressure measurement of the impact of higher compressor exit velocities and split diffuser designs. CFD will also be used to model the flow field within the compressor diffuser/combustor wrapper volume to evaluate potential problems and likely solutions.

\section{Progress Since the Last Reporting Period}

In this Quarter, testing to determine which style of diffuser would be used in the ATS machine was completed.

The selection criterion for choosing the best design was based on the diffuser's ability to deliver uniform cooling flow to the combustion system and the absence of aerodynamic problems.

Concurrent with the diffuser fabrication, the test facility is being readied for the diffuser pressure recovery tests. Flow profilers to make the inlet flow to the diffuser look like the range of flows that would come out of a typical compressor have been designed and submitted for fabrication. Thirty additional pressure measurement ports have been added to the facility to handle the diffuser wall static tap measurements. A total pressure probe and traversing mechanism has been added to determine both the diffuser inlet and exit velocity profiles.

\section{Plans for the Next Reporting Period}

The pressure recovery performance of the new diffuser will be determined.

A final report will be written. 


\section{Task 8.7.12 - Nozzle Cascade Preliminary Test and Facility Qualification}

\section{Objective}

Characterize the combustor exit conditions in both diffusion and premix combustor modes by testing with a nozzle box installed in place of the test nozzle airfoils, establish the Mach number distributions within the test passages to allow interpretation of heat transfer data to be obtained in the subseuqne test, and determine internal cooling circuitry conditions and airfoil metal temperature distributions by testing with extensive instrumentation with airfoils in place. This work is in preparation for the final LCF test of the Build 1 cascade.

The rig will be designed, constructed, and checked out during this phase of the program.

\section{Test Facility}

In order to evaluate the critical design assumptions for the first-stage nozzle system, a cascade test will be performed in the Nozzle Cascade Test Stand at the GEAE Laboratory in Evendale. This test will validate nozzle life prediction methodology and demonstrate the validity of the design concept. The Nozzle Cascade tests will validate the internal heat transfer and flow circuitry assumptions for steam cooling under actual machine conditions.

The nozzle Cascade Test Stand employs a full-size combustor, a transition piece, and a first-stage nozzle segment with three passages. The three passages are created by two actual nozzle segments and two end walls. The assembly is encapsulated in a $288 \mathrm{~cm}(90$ in.) diameter pressure vessel with the cascade efflux being discharged to a water quench through a $106 \mathrm{~cm}$ (42 in.) diameter duct. The combustor, transition piece, and nozzle segments are actual engine parts with the interfaces to the test facility mounting reflecting the engine configuration.

\section{Progress}

Under Task 1, Initial Build-up/Checkout/Combustor Mapping, an amendment was provided to the ATS Phase II NEPA report covering operation in this facility. Forty hours of combustion testing was completed, up to present facility pressure and flow limits. Transfers from diffusion mode to premixed mode and back were accomplished with no indication of adverse dynamic activity. Combustor operation was excellent over the entire range of testing. Test facility and test hardware operation at the conditions to be utilized for the LCF test cycle was successfully demonstrated during this phase of testing. The balance of the Task I Checkout and Mapping will be completed after installation and checkout of the Nuovo Pignone compressors has been completed.

Under Task 2, Cascade Pressure Tap Testing, all necessary testing was completed. Pressure distribution data were obtained over a variety of power settings, and showed excellent agreement with pre-test predictions. 
The heat transfer cascade to be tested in Task 3 has been delayed, and activity in support of this effort has been limited to pre-test planning. This testing is expected to be conducted during the first quarter of 1996.

No activity related to Task 4, LCF Cascade Test, has been initiated to date. This testing will follow the heat transfer testing in Task 3.

Under Task 5, Support Tooling and Equipment, the slave hardware required to support the steam cooling and slave water system for the cascade vehicle were designed and procured. This equipment has been functionally tested up to design conditions and has demonstrated satisfactory operation.

\section{Plans For Next Quarter}

The Phase 2 portion of this task has been completed, and a Topical Report will be written. 